\title{
Positron plasma control techniques for the production of cold antihydrogen
}

R. Funakoshi, ${ }^{1}$ M. Amoretti, ${ }^{2, *}$ G. Bonomi, ${ }^{3,4}$ P. D. Bowe, ${ }^{5}$ C. Canali, ${ }^{2,6}$ C. Carraro, ${ }^{2,6}$ C. L. Cesar, ${ }^{7}$ M. Charlton, ${ }^{8}$ M. Doser, ${ }^{9}$ A. Fontana, ${ }^{4,10}$ M. C. Fujiwara, ${ }^{11,12}$ P. Genova, ${ }^{4,10}$ J. S. Hangst, ${ }^{5}$ R. S. Hayano, ${ }^{1}$ L. V. Jørgensen, ${ }^{8}$ A. Kellerbauer, ${ }^{9} *$ V. Lagomarsino ${ }^{2,6}{ }^{2}$ R. Landua, ${ }^{9}$ E. Lodi Rizzini, ${ }_{13}^{1314}$ M. Macrì ${ }^{2}$ N. Madsen, ${ }^{5}$ G. Manuzio, ${ }^{2,6}$ D. Mitchard, ${ }^{8}$ P. Montagna, ${ }^{4,10}$ L. G. C. Posada, ${ }^{15}$ A. Rotondi, ${ }^{4,10}$ G. Testera, ${ }^{2}$ A. Variola, ${ }^{2}$ L. Venturelli, ${ }^{13,14}$ D. P. van der Werf, ${ }^{8}$ Y. Yamazaki, ${ }^{12}$ and N. Zurlo ${ }^{13,14}$

(ATHENA Collaboration)

${ }^{1}$ Department of Physics, University of Tokyo, Tokyo 113-0033, Japan

${ }^{2}$ Istituto Nazionale di Fisica Nucleare, Sezione di Genova, 16146 Genova, Italy

${ }^{3}$ Dipartimento di Ingegneria Meccanica, Università di Brescia, 25123 Brescia, Italy

${ }^{4}$ Dipartimento di Fisica Nucleare e Teorica, Università di Pavia, 27100 Pavia, Italy

${ }^{5}$ Department of Physics and Astronomy, University of Aarhus, 8000 Aarhus C, Denmark

${ }^{6}$ Dipartimento di Fisica, Università di Genova, 16146 Genova, Italy

${ }^{7}$ Instituto de Fisica, Universidade do Brasil, Cx Postal 68528, Rio de Janeiro 21941-972, Brazil

${ }^{8}$ Department of Physics, University of Wales Swansea, Swansea SA2 8PP, United Kingdom

${ }^{9}$ PH Department, CERN, 1211 Geneva 23, Switzerland

${ }^{10}$ Istituto Nazionale di Fisica Nucleare, Sezione di Pavia, 27100 Pavia, Italy

${ }^{11}$ TRIUMF, 4004 Wesbrook Mall Vancouver, BC V6T 2A3, Canada

${ }^{12}$ Atomic Physics Laboratory, RIKEN, Saitama 351-0198, Japan

${ }^{13}$ Dipartimento di Chimica e Fisica per l'Ingegneria e per i Materiali, Università di Brescia, 25133 Brescia, Italy

${ }^{14}$ Istituto Nazionale di Fisica Nucleare, Gruppo Collegato di Brescia, 25133 Brescia, Italy

${ }^{15}$ Department of Physics, University of Tokyo, Tokyo 153-8902, Japan)

(Received 22 April 2007; published 19 July 2007)

\begin{abstract}
An observation of a clear dependence of antihydrogen production on positron plasma shapes is reported. For this purpose a plasma control method has been developed combining the plasma rotating-wall technique with a mode diagnostic system. With the help of real-time and nondestructive observations, the rotating-wall parameters have been optimized. The positron plasma can be manipulated into a wide range of shapes (aspect ratio $6.5 \leqslant \alpha \leqq 80)$ and densities $\left(1.5 \times 10^{8} \leqslant n \lesssim 7 \times 10^{9} \mathrm{~cm}^{-3}\right)$ within a short duration $(25 \mathrm{~s})$ compatible with the ATHENA antihydrogen production cycle.
\end{abstract}

DOI: 10.1103/PhysRevA.76.012713 PACS number(s): 34.80.Lx, 36.10.-k, 52.27.Jt, 52.20.Hv

\section{INTRODUCTION}

In 2002 two experiments at CERN, ATHENA [1], and ATRAP [2], reported the production of cold antihydrogen $(\overline{\mathrm{H}})$ by mixing antiprotons $(\bar{p})$ and positrons $\left(e^{+}\right)$at low temperature in a nested Penning trap [3]. A major future goal of this field is the creation of a sample of trapped cold $\overline{\mathrm{H}}$ to facilitate a class of entirely new and crucial experiments on antihydrogen spectroscopy and perhaps tests of antimatter gravity.

The ATHENA experiment produced copious amounts of $\overline{\mathrm{H}}$ by merging $\bar{p}$ and $e^{+}$in a cryogenic $(15 \mathrm{~K})$ and ultrahighvacuum $\left(<10^{-12}\right.$ mbar $)$ environment, where the tens of millions of $e^{+}$which were typically utilized were dense enough to be in the plasma regime [1].

The production of $\overline{\mathrm{H}}$ by merging $e^{+}$and $\bar{p}$ is analogous to the production of hydrogen $(\mathrm{H})$ via electron $\left(e^{-}\right)$-proton $(p)$ interactions. The latter has been studied over the years, mainly at high temperatures, where astrophysical plasmas and the attendant physics of recombination are of interest.

*Present address: Max Planck Institute for Nuclear Physics, Postfach 103980, 69029 Heidelberg, Germany.
More recently, recombination of $e^{-}-p$ has been studied in storage rings, or in merged beams. This work has been reviewed elsewhere $[4,5]$. To our knowledge, there have been no studies of $\mathrm{H}$ formation from low energy $e^{-}$and $p$ in a Penning trap, though simultaneous confinement of both species in a combined Penning-Paul trap has been reported [6] as have studies of cooling of $p$ by a trapped $e^{-}$cloud [7].

The processes contributing to the production of $\overline{\mathrm{H}}$ are thought to be the radiative two-body and the collisional three-body reactions. The latter is a multistep process which is sensitive to the trap geometry and to the plasma characteristics. In principle the rates of these two processes have different dependencies on the temperature $T$ and density $n$ of the $e^{+}$plasma; for the two-body case the rate is expected to scale as $T^{-0.63}$ and $n$, whereas a trend of $T^{-9 / 2}$ and $n^{2}$ is anticipated for the simplest three-body process $[4,8-10]$. Experimentally, ATHENA found that by increasing the plasma temperature the fall-off in $\overline{\mathrm{H}}$ yield is slow enough that it is still measurable at room temperature. However, the trend was not easily explainable in terms of the two-body or threebody formation, since no simple $T$ power law fitted the data [11]. However, detailed Monte Carlo simulations [12] have revealed that three-body capture is as multistep process as the $\bar{p}$ repeatedly traverse the $e^{+}$cloud [13]. Thus, the properties of the latter (e.g., temperature, number, density, and 
shape) are important parameters. As such, detailed interpretation of the $\overline{\mathrm{H}}$ production experiments remains open to further theoretical and experimental investigations.

In this paper we report the experimental observation of clear differences in the $\overline{\mathrm{H}}$ production signals for three distinct types of $e^{+}$plasma shape. Since the ATHENA $\overline{\mathrm{H}}$ production cycle has very limited idle time available, rapid plasma manipulation methods have been developed using a combination of the plasma-mode system $[14,15]$ and the rotating-wall technique [16-18]. The plasma-mode system provides a nondestructive and real-time observation of the plasma conditions, allowing the optimization of the rotating-wall parameters for rapid compression or expansion of the positron plasma. After optimization, the rotating-wall was inserted into the ATHENA $\overline{\mathrm{H}}$ production cycle, and the resulting $\overline{\mathrm{H}}$ was monitored using a special imaging detector [19-21] as the $e^{+}$plasma shape was changed.

\section{PRINCIPLES OF THE PLASMA TECHNIQUES}

\section{A. Plasma-mode system}

The plasma-mode system of ATHENA [14,15] yields values for the plasma density, length, radius, and total particle number by exploiting the low-order oscillation modes. Based on cold fluid theory [22], for a strongly magnetized plasma, the dispersion relation is approximated by

$$
\epsilon_{3}-\frac{k_{2}}{k_{1}} \frac{P_{l}\left(k_{1}\right) Q_{l}^{\prime}\left(k_{2}\right)}{P_{l}^{\prime}\left(k_{1}\right) Q_{l}\left(k_{2}\right)}=0,
$$

where $k_{1}=\alpha\left(\alpha^{2}-\epsilon_{3}\right)^{-1 / 2}, k_{2}=\alpha\left(\alpha^{2}-1\right)^{-1 / 2}, \epsilon_{3}=1-\omega_{p}^{2} / \omega_{l}^{2}, \omega_{p}$ $=\left[4 \pi n(\alpha) e^{2} / m\right]^{1 / 2}$ is the the plasma frequency, $\alpha$ is the aspect ratio (the semimajor axis $z_{p}$ divided by its semiminor analog $r_{p}$ ) of the plasma and $n(\alpha)$ its density. $P_{l}$ and $Q_{l}$ are Legendre functions of the first and second kind, and $l$ is the azimuthal quantum number [23] and the primes denote the relevant single differentials. The solution to Eq. (1) for $\omega_{l}$ gives the $l$ th mode frequency; the value with $l=1(2)$ corresponds to the first (second) plasma mode frequency, or the dipole mode (quadrupole mode) frequency. Furthermore $\alpha$ and $n(\alpha)$ are related to the axial frequency $\omega_{1}$ through the equation [14]

$$
\frac{\omega_{1}^{2}}{\omega_{p}^{2}}=\frac{1}{\alpha^{2}-1} Q_{1}\left(k_{2}\right)
$$

From Eqs. (1) and (2), the relationship between $\omega_{1}, \omega_{2}$ and $\alpha$ can be written as

$$
\frac{\omega_{2}^{2}}{\omega_{1}^{2}}=\frac{\alpha^{2}-1}{Q_{1}\left(k_{2}\right)\left[1-\frac{k_{2}}{k_{1}} \frac{P_{2}\left(k_{1}\right) Q_{2}^{\prime}\left(k_{2}\right)}{P_{2}^{\prime}\left(k_{1}\right) Q_{2}\left(k_{2}\right)}\right]} .
$$

Thus, $\alpha$ can be obtained from Eq. (3) from the combination of observed dipole and quadrupole mode frequencies. Figure 1 shows the $\alpha$ dependence of the ratio of these two frequencies. In the range of large $\alpha$, the values obtained from the analysis of these modes become less reliable as small

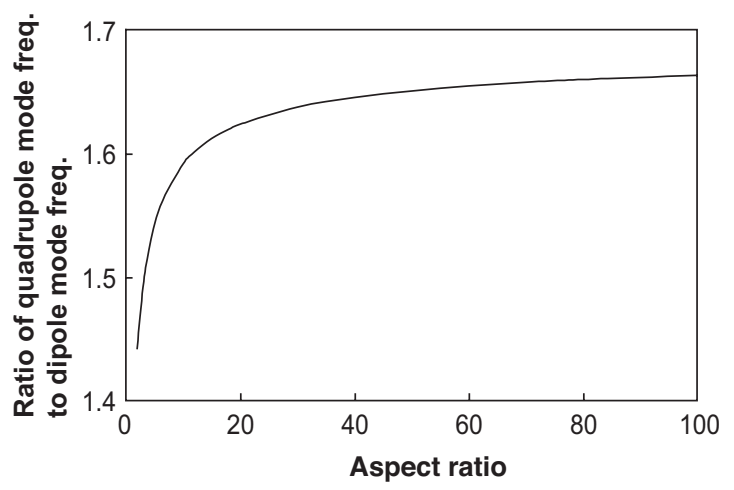

FIG. 1. The relationship between the mode frequencies (ratio of the quadrupole mode frequency to the dipole mode frequency) and the aspect ratio $\alpha(>1)$.

changes in the ratio between the mode frequencies result in large changes in $\alpha$.

\section{B. Rotating wall}

In Penning trap experiments, several groups have previously utilized the rotating-wall technique [16-18] in order to counter plasma expansion due to scattering of plasma particles with the background neutral gas and due to the presence of small static field errors which have been shown to limit plasma confinement time [24].

A rotating electric field applied to split electrodes can be used to alter the plasma shape. The plasma is compressed if the phase direction of the applied field is the same as that of the plasma radial motion and the frequency is higher than the plasma rotation frequency. On the contrary, the plasma is expanded if the direction of the electric field is counter propagating with respect to the plasma radial motion. In practice, a number of different techniques focused on differ-

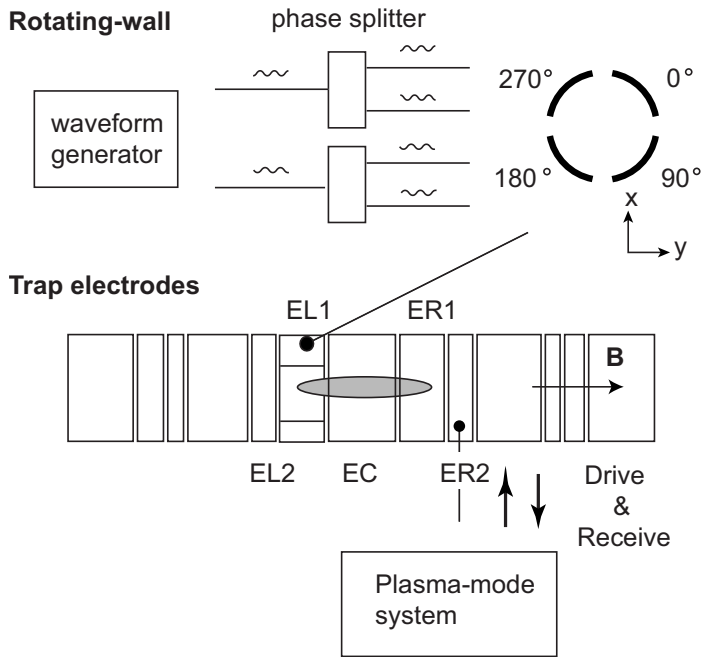

FIG. 2. Schematic diagram of the rotating-wall apparatus. The region from EL2 to ER2 is the central region of the nested Penning trap. The split electrode EL1 was connected to the rotating-wall modules and ER2 was connected to the plasma-mode system. 

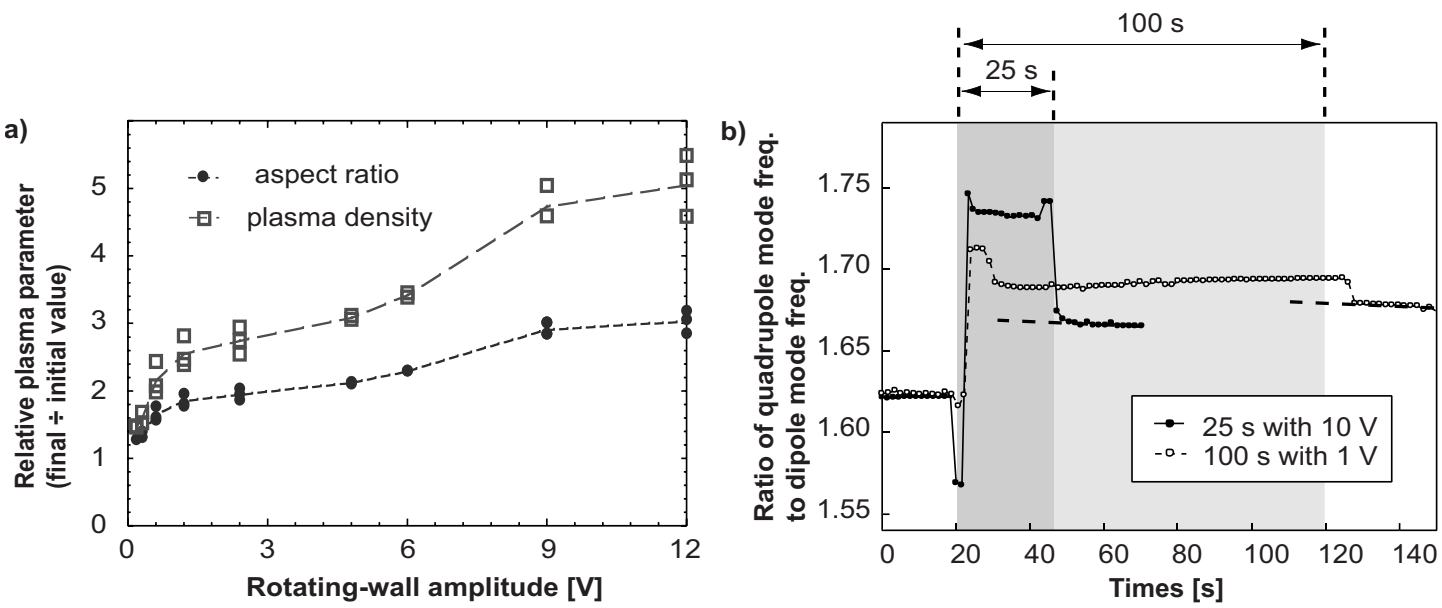

FIG. 3. (a) Effect of the rotating-wall on the aspect ratio and density of the positron plasma for various amplitudes. The lines are used to guide the eye. (b) Comparison of the effects of a $25 \mathrm{~s}$ wall with an amplitude of $10 \mathrm{~V}$ and a $100 \mathrm{~s}$ wall with $1 \mathrm{~V}$. The shaded areas represent the duration of the application of the rotating-wall voltage. After the rotating-wall voltage has been switched off, the signals on the plasma-mode system cannot be used to determine plasma mode frequencies for several seconds (see text). The data points on the dashed horizontal lines, however, are reliable and can be used to derive plasma parameters.

ent plasma radial motions have been developed to achieve plasma compression.

In the case of an ion plasma, the frequency of the rotating electric field has been applied slightly above the $\mathbf{E} \times \mathbf{B}$ frequency of the plasma [16]. In the case of $e^{-}\left(e^{+}\right)$plasmas, the applied frequencies have been much higher than the $\mathbf{E} \times \mathbf{B}$ rotation frequency, and some compression effects have been obtained when the applied frequencies coincided with those of the electrostatic mode generated by the internal radial motion of a spheroidal plasma, known as Trivelpiece-Gould modes $[17,18]$.

Recently a novel technique, dubbed strong-drive rotatingwall, has been reported for a system using a strong axial magnetic field for radial confinement [25]. Although the mechanism of this technique is not well understood, efficient compression can be achieved over a broad range of frequencies, without tuning to the plasma mode, by using an amplitude larger than that normally applied. Here the confinement provided by the magnetic field is strong enough to avoid losses caused by this large rotating-wall amplitude. In particular, an efficient rotating-wall compression was performed using a large amplitude $(1 \mathrm{~V})$ applied for $20 \mathrm{~s}$ in a $5 \mathrm{~T}$ magnetic field [25].

\section{PARAMETER OPTIMIZATION}

In order to optimize the rotating-wall to a duration short enough to be inserted into the ATHENA $\bar{H}$ production cycle, a large amplitude electric field to create a strong-drive rotating-wall was used. A schematic of the experimental setup is shown in Fig. 2. The $e^{+}$were confined in a Penning trap with a $1.25 \mathrm{~cm}$ electrode radius and a total length of $\sim 40 \mathrm{~cm}$, which was installed in a cryogenic $(15 \mathrm{~K})$ and ultrahigh-vacuum $\left(<10^{-12}\right.$ mbar $)$ environment. $7.5 \times 10^{7} e^{+}$ were loaded from the $e^{+}$accumulation system and held in a $50 \mathrm{~V}$ deep harmonic well located at the center of a nested
Penning trap. The trap consisted of 5 electrodes; electrodeleft (EL) 1 and 2, electrode-center (EC), and electrode-right (ER) 1 and 2. The plasma-mode system $[14,15]$ was connected to ER2. For the rotating wall, a wave function generator with a $180^{\circ}$ phase splitter was connected to the split electrode EL1 and used to make four sine-wave voltages, each with a phase difference of $90^{\circ}$. Thus the shape of the plasma was changed by applying a rotational electric field, and the resulting value of $\alpha$ was monitored using the plasmamode system.

Extensive surveys were made to optimize the performance in terms of frequency, amplitude, and duration of application [26]. Even using the strong-drive rotating-wall method, the rotating-wall previously used by ATHENA usually took $100 \mathrm{~s}$ to manipulate the $e^{+}$plasma in a $3 \mathrm{~T}$ magnetic field.

Since the ATHENA $\overline{\mathrm{H}}$ production cycle (see Sec. IV A) only allows $\sim 40 \mathrm{~s}$ for plasma manipulation, the rotatingwall amplitude was increased up to $12 \mathrm{~V}$, in order to improve its efficiency and thus shorten the application time. The drive frequency was set at $7 \mathrm{MHz}$. The results are presented in Fig. 3(a) which shows that the effect of the rotating-wall increases with the amplitude. It is important to note that no $e^{+}$loss occurred with the application of the rotating wall. This is indicated by the output of the plasmamode system by a comparison of signals before and after the application of the rotating-wall. This is corroborated by dumping the $e^{+}$at the end of the measurement onto a calibrated Faraday cup. Figure 3(b) shows a comparison between a rotating-wall of $10 \mathrm{~V}$ amplitude applied for $25 \mathrm{~s}$ and a $1 \mathrm{~V}$ wall applied for $100 \mathrm{~s}$. For these measurements a frequency of $7 \mathrm{MHz}$ was used. Since in both cases $\alpha$ from Eq. (3) (or Fig. 1) is difficult to estimate, here we simply show the ratio of the dipole mode frequency to that for the quadrupole for both. The shaded areas indicate the rotating-wall duration in which the mode frequencies cannot be used for $e^{+}$ plasma shape determination. This is because the quadrupole 
TABLE I. The parameters of three different types of $e^{+}$plasma achieved by the short duration ( $25 \mathrm{~s}$ ) rotating wall. The table shows typical values extracted using the plasma-mode diagnostics.

\begin{tabular}{lcccc}
\hline \hline & $\alpha$ & $r_{p}(\mathrm{~mm})$ & $z_{p}(\mathrm{~cm})$ & $n\left(\mathrm{~cm}^{-3}\right)$ \\
\hline compressed plasma & $\sim 80$ & $0.2-0.3$ & $\sim 2.2$ & $\sim 7 \times 10^{9}$ \\
standard plasma & 20 & 1.0 & 2.0 & $8 \times 10^{8}$ \\
expanded plasma & 6.5 & 2.5 & 1.6 & $1.5 \times 10^{8}$ \\
\hline \hline
\end{tabular}

mode frequency is sensitive to plasma temperature which is affected by heating induced by the rotating-wall voltage. Additionally, for several seconds after the rotating-wall has been removed, the plasma diagnostic cannot reliably measure the frequencies, probably due to the noise caused by the large amplitude of the rotating-wall. However, the data on the dashed lines in Fig. 3(b) can be used for $e^{+}$plasma shape determination. From these data it can be seen that the $25 \mathrm{~s}$ wall at $10 \mathrm{~V}$ can produce compression effects similar to the $100 \mathrm{~s} 1 \mathrm{~V}$ wall ( $\alpha$ is around 80 in both cases). Within this short duration the rotating-wall is able to control the $e^{+}$parameters within the range of desired shapes $(6.5 \leqslant \alpha \lesssim 80)$ and densities $\left(1.5 \times 10^{8} \leqslant n \lesssim 7 \times 10^{9} \mathrm{~cm}^{-3}\right)$. The parameters of typical $e^{+}$plasmas produced are summarized in Table I.

\section{APPLICATION TO ANTIHYDROGEN PRODUCTION}

\section{A. ATHENA antihydrogen production cycle}

The ATHENA apparatus consists of four parts [1,21]: (1) a $\bar{p}$ catching trap, (2) a $e^{+}$accumulator, (3) a mixing trap, and (4) an imaging detector. $\overline{\mathrm{H}}$ production is achieved by mixing of $\bar{p}$ and $e^{+}$in the mixing trap. When the $\bar{p}$ are mixed, $\overline{\mathrm{H}}$ annihilation events, which correspond to those $\overline{\mathrm{H}}$ atoms

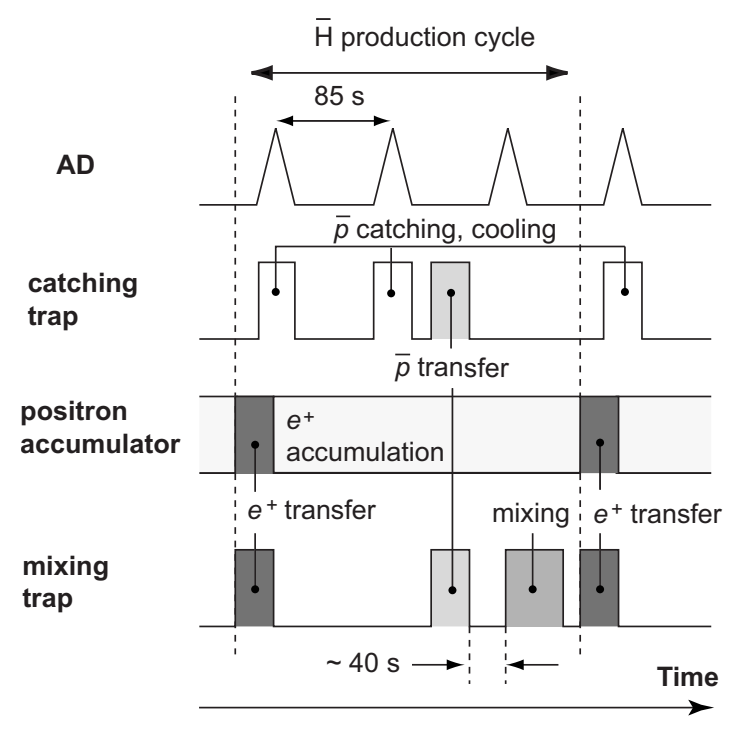

FIG. 4. Schematic illustration of the ATHENA $\bar{H}$ production cycle. Positrons are accumulated and transferred to the mixing trap every $\sim 200 \mathrm{~s}$ and $\bar{p}$ are accumulated by stacking $2 \mathrm{AD}$ shots and then mixed in the nested Penning trap for $60 \mathrm{~s}$. There is a $\sim 40 \mathrm{~s}$ pause between $\bar{p}$ transfer and the start of mixing. which survive the combined electric field of the trap and the $e^{+}$plasma, are identified using a purpose-built imaging detector. A schematic time line of the ATHENA $\overline{\mathrm{H}}$ production cycle is given in Fig. 4. Positrons from a radioactive ${ }^{22} \mathrm{Na}$ source $(1.4 \mathrm{GBq})$ are moderated and then cooled and collected in a $e^{+}$accumulator before being transferred to the mixing trap. Typically $7.5 \times 10^{7} e^{+}$are successfully transferred to the mixing region every $200 \mathrm{~s}$ [26]. Around $2.5 \times 10^{7} \bar{p}$ are provided by the Antiproton Decelerator (AD) with an energy of $5.3 \mathrm{MeV}$ every $85 \mathrm{~s}$ in a $200 \mathrm{~ns}$ wide pulse. After moderation by transmission through a thin aluminum foil at the entrance of the ATHENA apparatus, around $10^{4} \bar{p}$ are captured in the $\bar{p}$ catching trap using a pulsed electric field. They are then cooled by Coulomb scattering with preloaded $e^{-}$. This cooling continues until the $\bar{p}$ have reached the ambient temperature of the trap of about $15 \mathrm{~K}$. After stacking $2 \mathrm{AD}$ shots, the $\bar{p}$ are transferred from the catching trap to the mixing trap, and around 7000 of them are injected into the $e^{+}$plasma, which is located at the center of the nested Penning trap, and mixed for $60 \mathrm{~s}$.

$\mathrm{A} \sim 40 \mathrm{~s}$ pause in the $\overline{\mathrm{H}}$ production cycle (see Fig. 4) between $\bar{p}$ transfer and $\bar{p}$ injection (mixing) allows for the incorporation of a $25 \mathrm{~s}$ duration rotating wall for $e^{+}$plasma manipulation.

\section{B. Antihydrogen production and positron plasma shape}

Before $\bar{p}$ injection into the $e^{+}$, the rotating-wall was applied to the latter for $25 \mathrm{~s}$, followed by an additional pause of around $10 \mathrm{~s}$ to allow the $e^{+}$to cool back to ambient (which is presumably at, or near, the $15 \mathrm{~K}$ thermal environment of the traps) by the emission of synchrotron radiation. During mixing, data were collected by both the imaging detector and the plasma-mode system for $60 \mathrm{~s}$. This cycle was repeated for three different shapes of $e^{+}$plasmas: a compressed plasma $(\alpha \sim 80)$, a standard plasma $(\alpha=20)$, and an expanded plasma $(\alpha=6.5)$ (see Table I), and data were collected for each of them, for 20,14, and 38 cycles, respectively.

The trigger signal output from the imaging detector is shown in Fig. 5 for each type of plasma. The data are normalized to a single standard mixing cycle with $7000 \bar{p}$. Clear differences between the time dependence of the trigger signals for the three cases are observed. In the case of the standard plasma, the trigger signals decrease with time and two components can be seen in the time spectrum, a "prompt peak" and a "long tail." The prompt peak appears just after the $\bar{p}$ injection and the long tail appears later with a time constant of $10 \mathrm{~s}$. In the case of the compressed plasma, the prompt peak exceeds that of the standard plasma, and a new second broad peak appears several seconds after the start of mixing. In the case of the expanded plasma, the prompt peak disappears and only an enhanced long tail is present.

Subsequently, similar measurements were performed but with RF heating applied to the $e^{+}$. The frequency of the RF was tuned to be in resonance with the dipole mode, and had an applied amplitude of $12.6 \mathrm{mV}$. The temperature shift of the $e^{+}$was estimated to be $>3000 \mathrm{~K}$ [11]. Data for three cycles were accumulated for each plasma type and it was found that almost all the signal disappeared, due to suppres- 


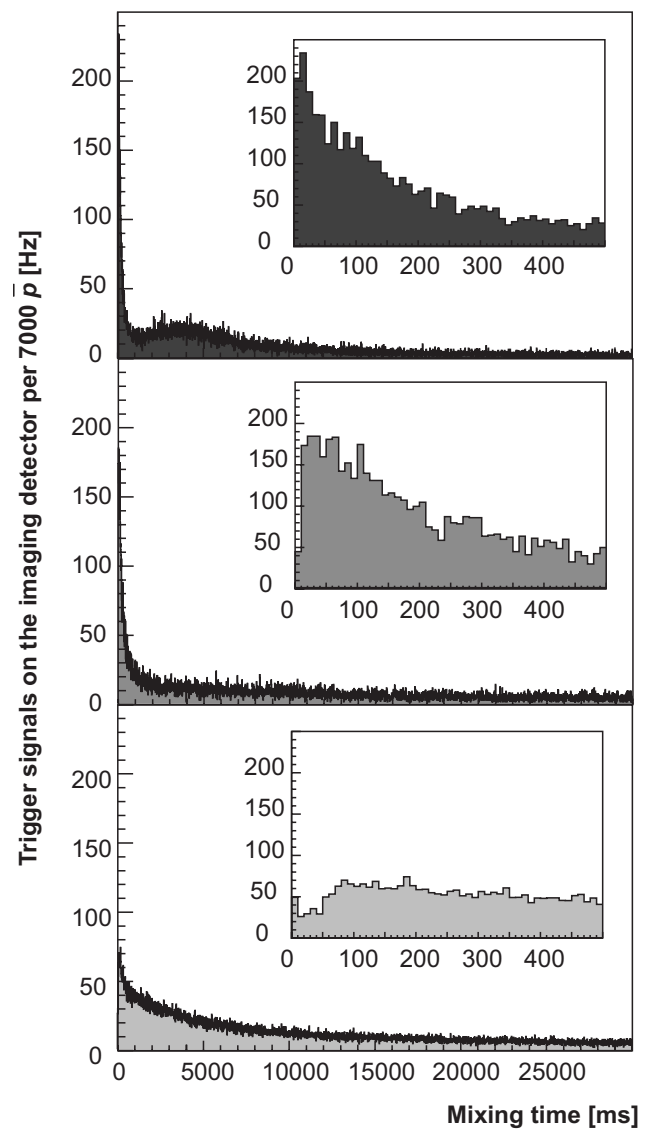

FIG. 5. Trigger signals on the imaging detector during mixing. From top to bottom: compressed plasma, standard plasma, and expanded plasma. Inset: the same for the first $500 \mathrm{~ms}$. All the data are normalized to a single standard mixing cycle with $7000 \bar{p}$.

sion of $\overline{\mathrm{H}}$ production. As our previous work has reported $[11,27]$, the trigger signal after subtraction of the RF heating background gives a reliable proxy for $\overline{\mathrm{H}}$ production. The time evolution of integrated number of triggers after subtraction of background for each type of plasma is presented in Fig. 6. This shows that, despite the fact that the $\overline{\mathrm{H}}$ yields for the compressed and standard plasmas are 4-5 times greater than that for the expanded plasma case in the first $100 \mathrm{~ms}$, eventually more $\overline{\mathrm{H}}$ is created for the last plasma. The yield for the expanded plasma exceeds that for the other two after about $1 \mathrm{~s}$ of mixing and eventually around twice as much $\overline{\mathrm{H}}$, bound sufficiently deeply to reach the wall of the trap and annihilate, is formed in this case. This is likely to be caused by more of the $\bar{p}$ cloud overlapping efficiently with the expanded $e^{+}$cloud.

\section{Study of the antiproton cooling efficiency}

In order to study the interaction between the $\bar{p}$ and the $e^{+}$ in more detail, we have also investigated the efficiency for cooling of the $\bar{p}$ by $e^{+}$on the plasma shape of the latter. A short mixing time of $100 \mathrm{~ms}$ [28] was set, after which the $\bar{p}$ were slowly released by changing the confinement potential in the three steps shown in Fig. 7(b). First (I) the potential

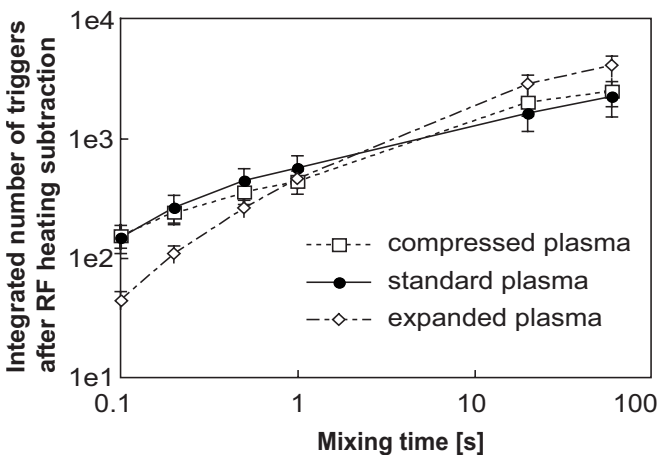

FIG. 6. The integrated number of $\overline{\mathrm{H}}$ triggers, after subtraction of background, scaled to a single standard mixing cycle with $7000 \bar{p}$. The samples are at times of $0.1,0.2,0.5,1,20$, and $60 \mathrm{~s}$ after the start of mixing.

barrier for the $\bar{p}$ of the left well (LW) is lowered to the same level as the $e^{+}$-well $\left(e^{+} \mathrm{W}\right)$ to release the high energy portion of the $\bar{p}$ from the whole trap. Then (II) the remaining potential barrier of LW is lowered to release the low energy $\bar{p}$ from this section. Finally (III) the right-well (RW) potential is raised to release the low energy $\bar{p}$ from there. The number of released $\bar{p}$ was monitored by external scintillators located outside the ATHENA main magnet [21]. The results are given in Fig. 7(a), where the $\bar{p}$ annihilation time-spectra for the three types of plasma are shown, and the numbers of triggers in each region are reported in Table. II.

The time evolution of the cooling process was previously studied (a more detailed description of a variety of cooling measurements can be found elsewhere [13]). There are two
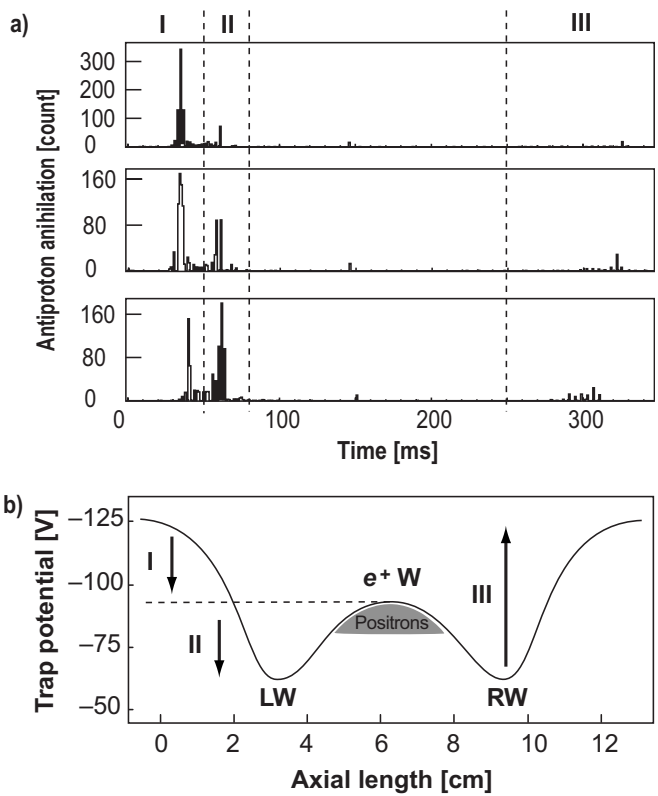

FIG. 7. (a) Time spectrum of the $\bar{p}$ annihilation signals. From top to bottom, the graphs are for the compressed plasma, the standard plasma, and the expanded plasma. $\bar{p}$ annihilations are monitored by the external scintillators [21] in this case. (b) The three step potential dump procedure (see text). The region labels are equivalent in both figures and in Table II. 
TABLE II. The number of $\bar{p}$ dumped after $100 \mathrm{~ms}$ of mixing. The regions correspond to those depicted in Fig. 7.

\begin{tabular}{lcccc}
\hline \hline & region I & region II & region III & total \\
\hline compressed & 704 & 147 & 28 & 879 \\
plasma & $(80.1 \pm 2.7 \%)$ & $(16.7 \pm 2.5 \%)$ & $(3.2 \pm 1.1 \%)$ & $(100 \%)$ \\
standard & 668 & 270 & 61 & 999 \\
plasma & $(66.9 \pm 3.0 \%)$ & $(27.0 \pm 2.8 \%)$ & $(6.1 \pm 1.5 \%)$ & $(100 \%)$ \\
expanded & 435 & 639 & 109 & 1183 \\
plasma & $(36.8 \pm 2.8 \%)$ & $(54.0 \pm 2.9 \%)$ & $(9.2 \pm 1.7 \%)$ & $(100 \%)$ \\
\hline \hline
\end{tabular}

different situations which govern $\bar{p}$ cooling by $e^{+}$in the nested Penning trap. Those $\bar{p}$ which initially make perfect radial overlap with the $e^{+}$are cooled within $\sim 10 \mathrm{~ms}$, and are separated into LW or RW after a few $100 \mathrm{~ms}$ of mixing. The remaining $\bar{p}$ are cooled and are axially separated more slowly with a time constant of $\sim 10 \mathrm{~s}$. The fractional number of $\bar{p}$ in region III indicates that little axial separation occurred in the first $100 \mathrm{~ms}$ of mixing in all the cases.

\section{Discussion}

During the first $100 \mathrm{~ms}$ of mixing, the $\bar{p}$ and $e^{+}$interact with each other many times, and form $\overline{\mathrm{H}}$ when their relative velocity becomes low enough. Antiprotons residing in regions II and III have overlapped with the $e^{+}$plasma and have been cooled to a low energy, but have not formed $\overline{\mathrm{H}}$ bound deeply enough to survive the electric field of the $e^{+}$plasma and the trap. Thus the probability of being in regions II and III, $P_{\mathrm{II}, \mathrm{III}}$ can be written as

$$
P_{\mathrm{II}, \mathrm{III}}=P_{\mathrm{oc}}\left(1-P_{\mathrm{H}}^{-}\right),
$$

where $P_{\mathrm{H}}^{-}$and $P_{\mathrm{oc}}$ are the probabilities of $\overline{\mathrm{H}}$ formation without electric field reionization and of overlap and cooling ready for $\overline{\mathrm{H}}$ formation, respectively. $P_{\mathrm{II}, \mathrm{III}}$ is obtained as the number of $\bar{p}$ in regions II and III divided by the total number of $\bar{p}$.

Meanwhile the probability to trigger the detector

$$
P_{\text {det }}=P_{\mathrm{oc}} P_{\mathrm{H}}^{-},
$$

is given as the number of triggers in the first $100 \mathrm{~ms}$ (see Fig. 6) divided by the total number of $\bar{p}$ in one mixing cycle.

Assuming a $\bar{p}$ number per mixing cycle of 7000 , which is a typical value in ATHENA, from Eq. (4) and Eq. (5) we obtain;

compressed plasma: $P_{\mathrm{oc}}=22.1 \pm 2.7 \%, P_{\mathrm{H}}=10.0 \pm 2.8 \%$,

$$
\text { standard plasma: } P_{\mathrm{oc}}=35.2 \pm 3.0 \%, \quad P_{\mathrm{H}}^{-}=6.0 \pm 1.9 \%,
$$

expanded plasma: $P_{\mathrm{oc}}=63.8 \pm 2.8 \%, \quad P_{\overline{\mathrm{H}}}=1.0 \pm 0.3 \%$.

The uncertainties given above have been increased over those due purely to statistics to allow for the small number of triggers caused by radial $\bar{p}$ losses [20] and the production of antiprotonic hydrogen [29]. The triggers from these effects combine to less than $20 \%$ of the total triggers in the first $100 \mathrm{~ms}$ of mixing. These results lead to the following observations.

(1) From Fig. 5 it can be seen that the rise time of the trigger signals depends upon the shape, and therefore the density, of the $e^{+}$plasma. The data are not in a suitable form to allow reliable estimates of the $\bar{p}$ cooling times to be extracted, particularly given that the latter pass in and out of the $e^{+}$plasma as they cool. However, the expected trend, whereby the time taken for the $\overline{\mathrm{H}}$ annihilation rate to peak decreases as the density is increased, is clearly observed. Unfortunately time spectra such as those in Fig. 7(a) are not available for mixing times shorter than $100 \mathrm{~ms}$.

(2) The values for $P_{\text {oc }}$ given above show a factor of around three increase for the expanded plasma when compared to the compressed plasma case. At first glance this is counter-intuitive, since $P_{\text {oc }}$ should be proportional to the product of the $e^{+}$plasma area and its density. Thus, if it is assumed that the $\bar{p}$ are uniformly distributed across the $e^{+}$, $P_{\mathrm{oc}}$ should be independent of $r_{p}$, which, from the $r_{p}$ values given in Table I, it clearly is not. This apparent contradiction is due to the fact that $P_{\mathrm{oc}}$ is extracted from analysis of the first 100 ms mixing. However, inspection of Fig. 5 shows that, for both the compressed plasma and standard plasma, the $\overline{\mathrm{H}}$ annihilation rate has already peaked by this time, such that cooling has occurred over a much shorter time scale.

(3) The $P_{\overline{\mathrm{H}}}$ values extracted from the analysis of the first $100 \mathrm{~ms}$ of events behave qualitatively as expected, with an order of magnitude increase in results for the compressed plasma relative to the expanded plasma.

Concerning the third item, the naive expectation is, as discussed briefly in Sec. I, that if the collisional three-body reaction dominates, and the $\bar{p}$ remain in a steady-state inside the $e^{+}$plasma, then the $\overline{\mathrm{H}}$ formation rate should vary as $n^{2}$. This would predict a 2000 -fold increase in $\overline{\mathrm{H}}$ formation rate for the compressed plasma when compared to the expanded plasma case.

Experimental work by ATHENA [11,30] has, however, suggested that $\overline{\mathrm{H}}$ is formed long before the $\bar{p}$ and $e^{+}$reach thermal equilibrium, such that the steady-state approach is not valid. Furthermore, it has been demonstrated by detailed simulations [12] that the multi-step, arrested, nature of the $\overline{\mathrm{H}}$ formation process, as the $\bar{p}$ pass to-and-fro in the $e^{+}$plasma, strongly modifies the outcome. As an example, it was found that the resulting $\overline{\mathrm{H}}$ atoms have much lower binding energies than would be expected from a steady-state situation. Thus, the $\overline{\mathrm{H}}$ atoms observed using the imaging detector (which forms the basis of our estimate of $P_{\mathrm{H}}^{-}$) are only a subset of those formed, namely those bound deeply enough to survive the ambient electric fields and then annihilate on the trap walls.

It is tempting to attempt to correct for this by estimating the maximum plasma electric field encountered by the nascent $\overline{\mathrm{H}}$ for each plasma type, and to use the scaling law found by Pohl et al. [31] to correct the probability for survival; i.e., to scale $P_{\overline{\mathrm{H}}}$. Since for each type of plasma $z_{p}$ $\gg r_{p}$, we can approximate the maximum plasma electric field as $E_{\max }=n e r_{p} / 2 \epsilon_{0}$. The values of $E_{\max }$ for each type of 
TABLE III. Electric field, $\overline{\mathrm{H}}$ binding energies, density, and $\overline{\mathrm{H}}$ formation probability parameters for the three different $e^{+}$plasmas. See text for details.

\begin{tabular}{lccccc}
\hline \hline & $\begin{array}{c}E_{\max } \\
\left(\mathrm{V} \mathrm{cm}^{-1}\right)\end{array}$ & $\begin{array}{c}\mathrm{BE} \\
(\mathrm{meV})\end{array}$ & $\begin{array}{c}n_{\mathrm{rel}}^{2} ; \\
n_{\mathrm{rel}}^{2}\end{array}$ & $\begin{array}{c}E_{\max }^{\mathrm{rel}} ; \\
\left(E_{\max }^{\mathrm{rel}}\right)^{2}\end{array}$ & $\begin{array}{c}P_{-}^{\mathrm{rel}} ; \\
P_{\mathrm{H}}^{\mathrm{rel}}\left(E_{\mathrm{max}}^{\mathrm{rel}}\right)^{2}\end{array}$ \\
\hline $\begin{array}{l}\text { compressed } \\
\text { plasma }\end{array}$ & 160 & 4.8 & $47 ;$ & $4.7 ;$ & $10 ;$ \\
standard & 72 & 3.2 & $5.3 ;$ & $2.1 ;$ & $6 ;$ \\
plasma & & & 28.1 & 4.4 & 26.4 \\
expanded & 34 & 2.2 & $1 ;$ & $1 ;$ & $1 ;$ \\
plasma & & & 1 & 1 & 1 \\
\hline \hline
\end{tabular}

plasma are given in Table III, along with the implied minimum binding energy $(\mathrm{BE})$ of the $\overline{\mathrm{H}}$ calculated from $\mathrm{BE}$ $=0.38\left(E_{\max }\right)^{1 / 2}\left(\mathrm{meV}\right.$ with $E_{\max }$ in $\left.\mathrm{Vcm}^{-1}\right)$ [32]. In Table III the plasma densities and electric fields and the $P_{\overline{\mathrm{H}}}$ are given relative to unity for the expanded plasma case as $n_{\text {rel }}, E_{\mathrm{max}}^{\text {rel }}$, and $P_{\overline{\mathrm{H}}}^{\mathrm{rel}}$, respectively.

Following the observations of Pohl et al. [31] who found that the probability of an $\overline{\mathrm{H}}$ atom surviving an electric field $E$ varied as $E^{-2}$, we construct the scaled parameter $P_{\overline{\mathrm{H}}}^{\mathrm{rel}}\left(E_{\max }^{\mathrm{rel}}\right)^{2}$. These values are given in the final column of Table III and should be compared to the scaled values of $n_{\text {rel }}^{2}$. Remarkably, the corrected $P_{\mathrm{H}}$ for the standard plasma scales as $n_{\text {rel }}^{2}$, though that for the compressed plasma case is an order of magnitude below the expectation. A possible reason for this is discussed below.

From Fig. 5, the annihilation time spectrum for the compressed plasma has a feature which is not present in the data for the other two cases, identified above as a second, broad peak which appears several seconds after the start of mixing. Observation of this effect, together with the large promptpeak shortly after mixing begins, allows us to confirm our previous interpretation [13] of the long-tail. In [13] this effect was ascribed to more gradual $\overline{\mathrm{H}}$ formation due to overlap of initially radially separated $\bar{p}$ with the $e^{+}$plasma. This may be due to a gradual expansion of the $e^{+}$plasma throughout the mixing cycle, which has already been identified by ATHENA using the plasma modes diagnostic system $[14,15]$. Explicit data can be found in Ref. [33].

The expanded plasma probably overlaps most, if not all of the $\bar{p}$, and at this lowest density, no prompt peak is observed. Rather, $\overline{\mathrm{H}}$ formation proceeds throughout the mixing cycle at a reduced rate. That the origin of the $\overline{\mathrm{H}}$ events at long times in this case is distinct from the two narrower and more dense plasmas can be seen in Fig. 5 and in the integrated yields presented in Fig. 6. For the standard plasma case, the $e^{+}$ plasma is now narrower than the $\bar{p}$ cloud. The $\overline{\mathrm{H}}$ formation is peaked at early times due to the efficient interaction of the overlapping clouds, and this is gradually supplemented at long times by the expansion of the $e^{+}$into the initially radially separated $\bar{p}$. In the compressed plasma case, the dense $e^{+}$ cloud is now much smaller than the $\bar{p}$. Formation of $\overline{\mathrm{H}}$ by those $\bar{p}$ which overlap with this plasma is very rapid and effectively depletes the inner region of the $\bar{p}$ cloud. The second broad peak arises as $\overline{\mathrm{H}}$ formation begins to recover as the $e^{+}$expand gradually into the $\bar{p}$ located at larger radii.

The latter contention also allows a possible explanation of the order of magnitude difference between $n_{\text {rel }}^{2}$ and $P_{\mathrm{H}}^{\mathrm{rel}}\left(E_{\mathrm{max}}^{\mathrm{rel}}\right)^{2}$, as shown in Table III for the compressed plasma case. The yield of $\overline{\mathrm{H}}$ integrated over $100 \mathrm{~ms}$ is essentially saturated in this case such that it is not representative of the true rate of $\overline{\mathrm{H}}$ formation.

The results of these brief experiments with three different plasma types are indicative of some of the rich spectrum of physical effects which govern $\overline{\mathrm{H}}$ formation and detection in nested Penning traps. Since the latter are expected to be the main vehicle for future work in this area (see, e.g., Ref. [34]), further experiments and simulations are desirable to extend our understanding of the details of the $e^{+}-\bar{p}$ interaction dynamics.

\section{SUMMARY}

The rotating-wall technique is capable of reproducibly controlling the shape and density of positron plasmas used for antihydrogen formation experiments. We have performed an optimization procedure of the rotating-wall parameters with the help of the ATHENA non-destructive plasma modes diagnostic and have achieved a wide range of $e^{+}$plasma shapes $(6.5 \leqslant \alpha \lesssim 80)$ and densities $\left(1.5 \times 10^{8} \leqslant n \lesssim 7\right.$ $\times 10^{9} \mathrm{~cm}^{-3}$ ) using a $25 \mathrm{~s}$ rotating-wall under conditions in which a strong magnetic field of $3 \mathrm{~T}$ was used.

Subsequently, this optimized rotating-wall has been incorporated into the ATHENA antihydrogen production cycle and three different shapes of positron plasmas have been prepared for use during mixing of antiprotons and positrons. The imaging detector was used for antihydrogen production diagnosis and clear differences between these three positron plasma configurations were observed. These results show that the rotating-wall technique allows detailed control of the antihydrogen formation process and will prove invaluable in future experiments aimed at trapping antihydrogen [34].

\section{ACKNOWLEDGMENTS}

We acknowledge CERN's PS and AD crew for providing the excellent antiproton beam. This work was supported by INFN (Italy), FAPERJ (Brazil), MEXT (Japan), SNF (Switzerland), NSRC (Denmark), EPSRC (UK), and the European Union. 
[1] M. Amoretti et al., Nature (London) 419, 456 (2002).

[2] G. Gabrielse et al., Phys. Rev. Lett. 89, 213401 (2002).

[3] G. Gabrielse et al., Phys. Lett. A 129, 38 (1988).

[4] M. H. Holzscheiter and M. Charlton, Rep. Prog. Phys. 62, 1 (1999).

[5] A. Müller and A. Wolf, Hyperfine Interact. 109, 233 (1997).

[6] J. Walz, S. B. Ross, C. Zimmermann, L. Ricci, M. Prevedelli, and T. W. Hänsch, Phys. Rev. Lett. 75, 3257 (1995).

[7] D. S. Hall and G. Gabrielse, Phys. Rev. Lett. 77, 1962 (1996).

[8] H. A. Bethe and E. E. Salpeter, Quantum Mechanics of Oneand Two-Electron Systems (Springer, Berlin, 1957).

[9] P. Mansback and J. C. Keck, Phys. Rev. 181, 275 (1969).

[10] J. Stevefelt, J. Boulmer, and J.-F. Delpech, Phys. Rev. A 12, 1246 (1975).

[11] M. Amoretti et al., Phys. Lett. B 583, 59 (2004).

[12] F. Robicheaux, Phys. Rev. A 70, 022510 (2004).

[13] M. Amoretti et al., Phys. Lett. B 590, 133 (2004).

[14] M. Amoretti et al., Phys. Plasmas 10, 3056 (2003).

[15] M. Amoretti et al., Phys. Rev. Lett. 91, 055001 (2003).

[16] X.-P. Huang, F. Anderegg, E. M. Hollman, C. F. Driscoll, and T. M. O’Neil, Phys. Rev. Lett. 78, 875 (1997).

[17] F. Anderegg, E. M. Hollmann, and C. F. Driscoll, Phys. Rev. Lett. 81, 4875 (1998).

[18] R. G. Greaves and C. M. Surko, Phys. Rev. Lett. 85, 1883 (2000).

[19] C. Regenfus et al., Nucl. Instrum. Methods Phys. Res. A 501,
65 (2003)

[20] M. C. Fujiwara et al., Phys. Rev. Lett. 92, 065005 (2004).

[21] M. Amoretti et al., Nucl. Instrum. Methods Phys. Res. A 518, 679 (2004).

[22] D. H. E. Dubin, Phys. Rev. Lett. 66, 2076 (1991).

[23] M. D. Tinkle, R. G. Greaves, C. M. Surko, R. L. Spencer, and G. W. Mason Phys. Rev. Lett. 72, 352 (1994).

[24] J. Notte and J. Fajans, Phys. Plasmas 1, 1123 (1994).

[25] J. R. Danielson and C. M. Surko, Phys. Rev. Lett. 94, 035001 (2005).

[26] L. V. Jørgensen et al., Phys. Rev. Lett. 95, 025002 (2005).

[27] M. Amoretti et al., Phys. Lett. B 578, 23 (2004).

[28] Note that a mixing time of $10 \mathrm{~ms}$ was quoted in error in Ref. [13] for this work. Most of the data reported in Ref. [13] were taken using different $e^{+}$plasma parameters than the work described in the present report.

[29] N. Zurlo et al., Phys. Rev. Lett. 97, 153401 (2006).

[30] N. Madsen et al., Phys. Rev. Lett. 94, 033403 (2005).

[31] T. Pohl, H. R. Sadeghpour, and G. Gabrielse, Phys. Rev. Lett. 97, 143401 (2006).

[32] See, e.g., C. F. Driscoll, Phys. Rev. Lett. 92, 149303 (2004).

[33] M. Amoretti et al., in Non-Neutral Plasmas V, AIP Conf. Proc. No. 692, edited by M. Schauer et al. (AIP, Melville, 2003), p. 121.

[34] G. Andresen et al., Phys. Rev. Lett. 98, 023402 (2007). 